\title{
Effective Treatment of NSCLC with Surgery After Nivolumab Combined with Chemotherapy: A Case Report and Brief Review of the Literature
}

This article was published in the following Dove Press journal: OncoTargets and Therapy

\section{Liangchen Zhao \\ Luzhen $\mathrm{Li}$ \\ Ting Chen \\ Cantu Fang (D)}

Department of Oncology, Zhongshan Hospital Affiliated to Guangzhou TCM University, Zhongshan, People's Republic of China
Correspondence: Cantu Fang Zhongshan Hospital Affiliated to Guangzhou TCM University, Zhongshan, People's Republic of China Email 3568076269@qq.com
Introduction: Immuno checkpoint inhibitors (ICIs) including anti-PD-L1 antibody have shown certain therapeutic effects on various cancer types. Here, we reported a case of the patient with resectable non-small cell lung cancer (NSCLC) showing a complete response to nivolumab combined with chemotherapy.

Patient Information: A 66-year-old male was diagnosed with stage IIIA large cell lung cancer, cT2N2M0, who was considered impossible to have a tumor resection due to his right hilar node enlargement. The diameter of the neoplasms was $22 \mathrm{~mm}$, and the patient wanted to get the chance of surgery. Light of all, surgery was the only radical treatment option and therefore planned.

Interventions: After administering 2 cycles of nivolumab combined with paclitaxel and cisplatin, the second chest computed tomography (CT) after the first scanning revealed the tumor apparent shrinking and vascular compression was disappeared than before. We performed a right upper lobectomy and mediastinal lymph node dissection. Pathologically, we confirmed no large cell lung cancer cells in the resected lung specimen.

Outcomes: The follow-up showing that patient remains alive without recurrence to these days.

Conclusion: This case report may provide a clue to the future development of induction therapy using nivolumab and surgery. The combined treatment of nivolumab and chemotherapy is likely to be considered as an optional management of resectable NSCLC.

Keywords: non-small cell lung cancer, neoadjuvant immunotherapy, nivolumab, case report

\section{Background}

Lung cancer is the first leading cause of cancer-related death worldwide, with the highest morbidity. Patients with early and locally advanced lung cancer could prolong their lifespan by surgery, but to a certain degree, the effect of surgery is limited. Neoadjuvant therapy gives early and locally advanced lung cancer patients benefits, it helps downgrade tumors and even create surgery opportunities for patients who were initially unable to operate. In recent years, many trials have shown the results that in neoadjuvant therapy, immunotherapy is more effective and safer than chemotherapy, it contributes to prolong patients' survival. Herein, we reported a case of an elder man with locally advanced lung large cell carcinoma who underwent surgery after receiving neoadjuvant immunotherapy, and reviewed the application of nivolumab in NSCLC briefly. 


\section{Case Report}

A 66-year-old male was admitted to our hospital on June 13, 2019, due to "chest tightness and coughing for more than half a month", with a history of a lung mass on a positron emission tomography (PET) scan performed at another hospital. He had no relevant past medical or family history, nor had he taken any medication. He used to smoke for more than 30 years and had been kicked the habit for more than 10 years. At the time of admission, his physical examination showed that the lung sounds in the right upper lung field were weakened. In order to assess the quality of his lung, he underwent a computed tomography (CT) scan and found that the density of the irregular soft tissue in the anterior segment of the right upper lung lobe was about $22 \mathrm{~mm} \times 19 \mathrm{~mm}$, and there were multiple burr shadows on the edges (Figure 1). It suggested that
A

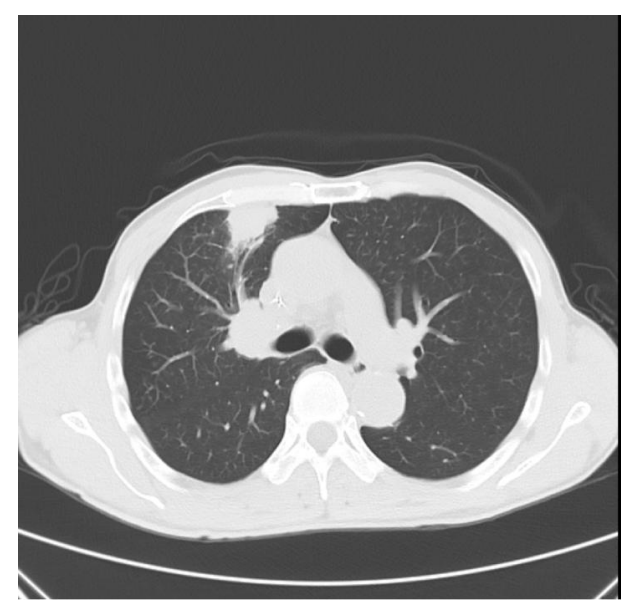

B

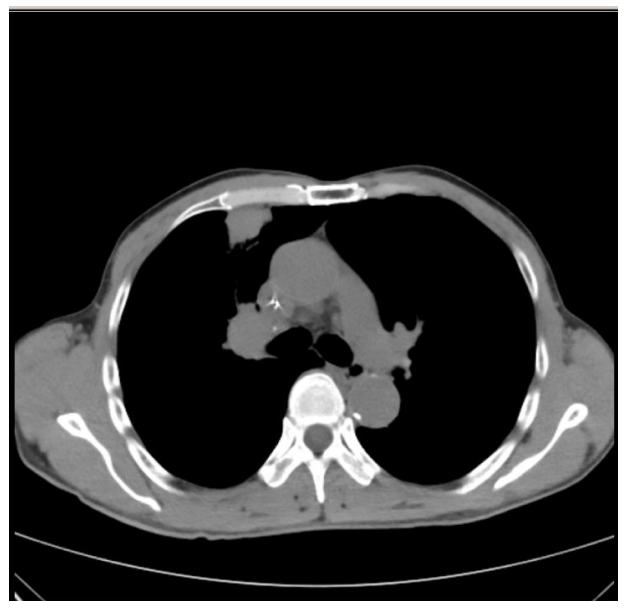

Figure I (A) Chest enhanced CT before treatment: an anterior segment of the right upper lobe with an irregular soft tissue density of approximately 22 mm $\times 19$ mm in shape. (B) Multiple burr shadows on the edges, and multiple enlarged lymph nodes in the right hilum.
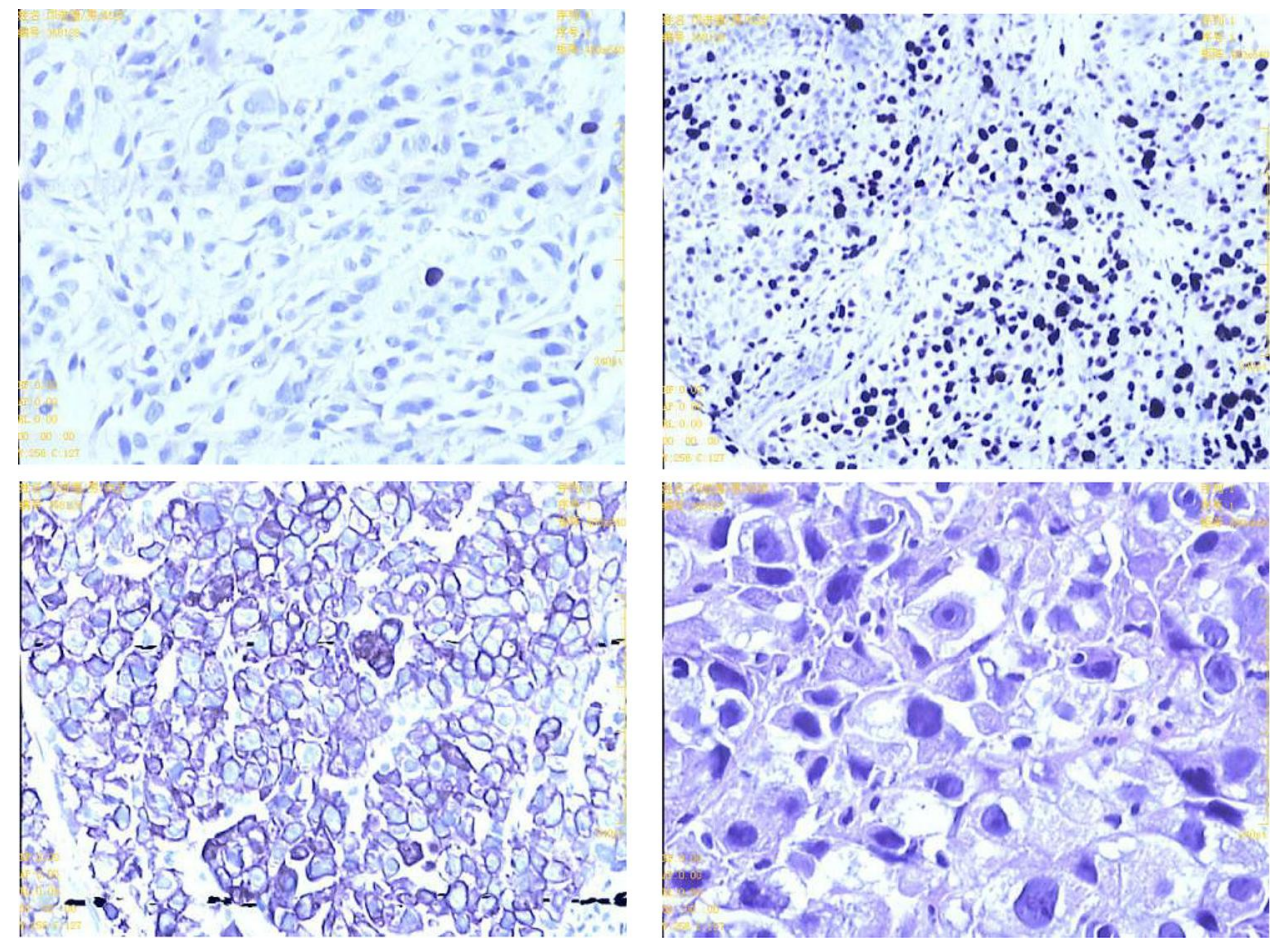

Figure 2 Right lung tumor biopsy specimen: malignant tumor of epithelial origin, prone to large cell carcinoma. Immunohistochemistry: CK (+), Ki67 (60\% +), P63 individual cells (+), TTF-I (-), Syn (-), CD56 (-), CgA (-). 
the right upper lobe lung cancer was complicated by hilar and mediastinal lymph node metastasis. Then, the patient underwent a fine needle aspiration biopsy of the right lung mass, the pathologic diagnosis was large cell carcinoma (Figure 2). Molecular analysis showed no epidermal growth factor receptor (EGFR), anaplastic lymphoma kinase (ALK) gene mutations, either. By direct sequencing, retrospective programmed death-ligand 1 (PD-L1) immunostaining showed high PD-L1 expression with a tumor proportion score, more than $50 \%$. Based on the examinations, a tentative diagnosis of lung cancer with T3N2M0 (stage IIIA, according to the seventh edition of TNM) was proposed. Given that the tumor was unresectable, the preferred treatment was sequential immunotherapy after simultaneous radiotherapy and chemotherapy. But the patient requested surgery strongly. After assessment by the multiple disciplinary team (MDT) in our hospital, we considered that the portal lymph nodes were swollen and had obvious adhesion with the right pulmonary artery and the right upper pulmonary vein, which could not be completely removed by surgery. According to the NADIM study updated by the 2019 ASCO annual meeting, ${ }^{1}$ and his genetic test results, it was feasible to perform neoadjuvant therapy with chemotherapy plus immunotherapy mode, to achieve the purpose of downgrading surgery. Then, he received 2 cycles of neoadjuvant chemotherapy of nivolumab+cisplatin-paclitaxel from July to August 2019 (actual usage: Paclitaxel 210mg + cisplatin $120 \mathrm{mg}+$ nivolumab $360 \mathrm{mg}$, q21d). During the two cycles of nivolumab treatment, the patient was diagnosed with moderate bone marrow suppression based on blood test. After 2 cycles of neoadjuvant therapy, the chest was rechecked with enhancement $\mathrm{CT}$. We observed that the area of the anterior segment of the right upper lobe of the lung was significantly reduced compared to the previous one. The maximum cross section was about $11 \mathrm{~mm} \times 7 \mathrm{~mm}$. The enlarged lymph nodes in the mediastinum and right hilar were smaller than before, and the compression of the adjacent pulmonary vessels was relieved (Figure 3). At this time, he was diagnosed with right upper lung cancer (large cell carcinoma) with right lung and mediastinal lymph node metastasis, cT2N2M0 stage IIIA. The patient's general condition was not very bad. He rejected the third cycle of chemotherapy+immunotherapy and requested surgery as soon as possible. After another MDT assessment, considering that the patient responded relatively well to the therapy, according to the response evaluation criteria for solid tumors (RECIST), the longest diameter of the target lesion was reduced by $50 \%$, and the efficacy evaluation was partial response (PR). Moreover, the pressure of blood vessels was relieved more than before, surgical treatment was feasible. Then, he was performed a right upper lobe anterior segmentectomy and mediastinal lymph node dissection. Pathology of the nodule confirmed a diagnosis of stage IIIA NSCLC (T2N2M0). It had been seen from the postoperative pathological section that the patient achieved pathologic complete response (pCR) after treatment, so no subsequent treatment was performed after the operation. After an uneventful recovery from surgery, he has been going to
A

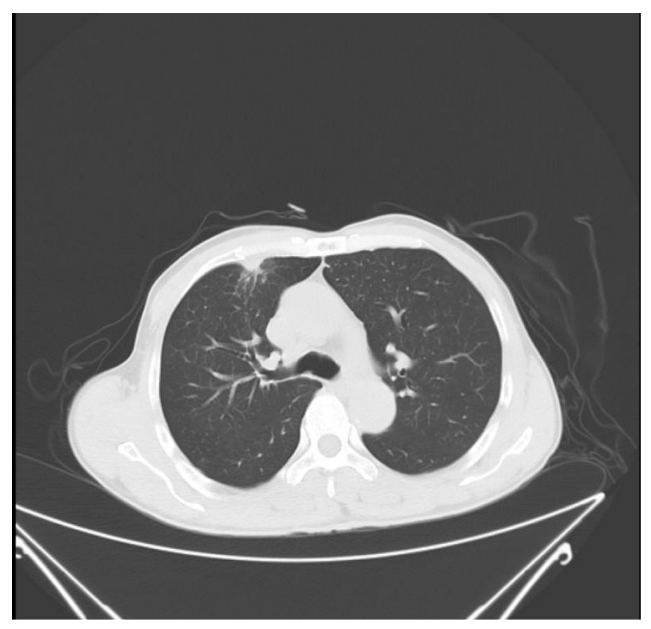

B

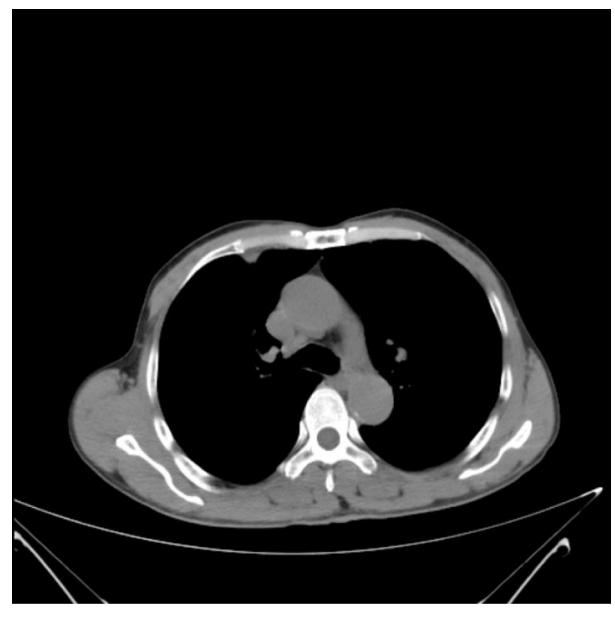

Figure 3 Chest enhanced CT after neoadjuvant immunotherapy: (A) The area of the anterior segment of the right upper lobe of the lung was significantly smaller than that of the anterior, with a maximum cross-section of about II mm $\times 7 \mathrm{~mm}$. (B) The mediastinal and right hilar swollen lymph nodes were smaller than before, and the adjacent pulmonary vessels were more compressed Before remission. 
the hospital for checkups regularly. Currently, the patient remains on oncological follow-up visits. At the last follow-up visit, in November 2020, the CT scan showed no disease recurrence.

\section{Discussion}

During a long time, surgery is usually considered as the treatment option for early disease. However, in stage III diseases, many patients have locally advanced or lymphatic metastasis at diagnosis, in which case, treatment is limited. Historically, patients with stage III NSCLC have poorer clinical outcomes, but recent advances in the treatment of adjuvant immune checkpoint blockade (ICB) have shown survival benefit, and recent data also show the benefit with adjuvant nivolumab. Immune checkpoint inhibitor therapy has changed the standard drug therapy for relapsed or advanced non-small cell lung cancer. With the rapid development of treatment methods, many studies have illustrated that immunotherapy has been extensively used in cancer treatment. Immunotherapy can alleviate the restriction of the immune system (PD-1 inhibitor) or cancer cell defense system (PD-L1 inhibitor), activates the body's own immune system, and enables tumor-specific $\mathrm{T}$ cells to better recognize and eliminate cancer cells and tumor tissue. Compared with chemotherapy and targeted drugs, the biggest feature of immunotherapy is not to blow out the cancer cells themselves, but to kill the cancer cells through the host's immune response, thereby returning the patient's own immune function to normal. Herein, we summarize the novel new therapies of nivolumab in NSCLC.

In many studies of the treatment of NSCLC, nivolumab's efficacy and safety are exciting. Patients with early, mid-term and partially resectable locally advanced lung cancer can be treated by surgical resection due to the limitations of the lesion. The combination of nivolumab and surgery could reduce the tumor stage, reduce the difficulty of surgery, and maximize the complete removal of tumor tissue, thereby helping to prolong the progression-free survival time of locally advanced patients.

Checkmate 159 study is the first trail to study immunoadjuvant therapy for NSCLC. ${ }^{2}$ The study included 21 cases of early-stage (I stage of -IIIA) patients, $86 \%$ of patients had a history of smoking. Before the operation, they received 2 cycles of nivolumab treatment. Of these 20 patients who received the surgery as scheduled, during the 1-year follow-up, 16 patients were still alive and had no tumor recurrence. At a follow-up of 18 months, the overall progression-free survival (PFS) rate of the patient was $73 \%$. At a follow-up of 30 months, 15 of 20 patients were disease-free and alive. The $24 \mathrm{~m}$ RFS rate was $69 \%$ (95\% CI: 51-93). ${ }^{3}$ In terms of safety, this study showed treatment-related adverse reactions were $23 \%$, only 1 case exceeded grade 3 .

The NADIM study aims to explore the efficacy of immunotherapy combined with chemotherapy for patients with stage IIIA NSCLC. ${ }^{1}$ The study admitted 46 patients with resectable tumor stage IIIA (N2, T4N0/N1), and EGFR/ALK negative. It was confirmed that 41 patients were performed surgery with $\mathrm{r} 0$ resection, 34 patients (83\%) achieved major pathological response (MPR), including $24(59 \%)$ of them were pCR, the World Conference on Lung Cancer (WCLC) 2019 reported that the 12-month PFS was 95.7\%, the 18-month PFS was $81 \%$, the median follow-up was 13.8 months, OS in the intent-to-treat population was $98 \%(95 \% \mathrm{CI}, 85-100)$ at 12 months and 91\% (95\% CI, 73-97) at 18 months, ${ }^{4}$ and with $90.2 \%$ of patients $(37 / 41)$ had staged decline. ${ }^{5}$ No surgical delay occurred. ${ }^{6}$ At present, other indicators of the study are still under observation. In the NADIM study, the pathological significant remission rate was significantly higher than similar data in chemotherapy and singleagent immunity studies, implementing the value-added benefit of $1+1>2$.

Checkmate 816 , which had met a primary endpoint of pathologic complete response (pCR) in resectable nonsmall cell lung cancer (NSCLC). In the trial, significantly more patients treated with nivolumab plus chemotherapy before surgery showed no evidence of cancer cells in their resected tissue compared to those treated with chemotherapy alone.

In addition to monotherapy and combined chemotherapy, dual immunotherapy is another new ideas. NEOSTAR is a Phase II study designed to evaluate the efficacy of nivolumab combined with Ipilimumab or neoadjuvant nivolumab in the treatment for stage-IIIA resectable NSCLC. Results of NEOSTAR showed that the total MPR rate of 37 evaluable patients was $30 \%$, of which the MPR rates of the nivolumab group and the combined group were $19 \%$ and $44 \%$, respectively. The objective response rate (ORR) of the intention-to-treat (ITT) population was $20 \%$, and the MPR response rates of the nivolumab group and the combination treatment group were $22 \%(4 / 23)$ and $19 \%(5 / 21)$, respectively. MPR rate was up to $78 \%$ among the patient who obtained complete response $(\mathrm{CR})$ or partial response $(\mathrm{PR})$. The study also 
Table I Clinical Researchers of Nivolumab in Neoadjuvant Immunotherapy with NSCLC

\begin{tabular}{|c|c|c|c|c|c|c|}
\hline Trails & NCT & $\begin{array}{l}\text { Primary } \\
\text { Outcome } \\
\text { Measures }\end{array}$ & $\begin{array}{l}\text { Secondary Outcome } \\
\text { Measure }\end{array}$ & $\begin{array}{l}\text { Estimated } \\
\text { Enrollment }\end{array}$ & Stage & $\begin{array}{l}\text { Estimated Primary } \\
\text { Completion Date }\end{array}$ \\
\hline Checkmate816 & 02998528 & EFS, pCR & OS, MPR, TTDM & 350 & $\mathrm{IB}(\mathrm{T}>4 \mathrm{~cm})-\mathrm{III} \mathrm{A}$ & $2020 / 4 / 8$ \\
\hline Checkmate77T & 04025879 & EFS & OS, pCR, MPR, SAEs, AEs & 452 & $\| \mathrm{A}(\mathrm{T}>4 \mathrm{~cm})-\mathrm{IIB}(\mathrm{T} 3 \mathrm{~N} 2)$ & $2023 / 5 / 24$ \\
\hline ANVIL & 02595944 & DFS, OS & AEs & 903 & IB $(T \geq 4 \mathrm{~cm})-$-IIIA & $2024 / 7 / 1$ \\
\hline NADIM II & 03838159 & $\mathrm{pCR}$ & - & 90 & IIIA-IIIB (T3N2) & $2022 / 3 / 15$ \\
\hline
\end{tabular}

Abbreviations: NSCLC, non small cell lung cancers; EFS, event-free survival; OS, overall survival; TTDM, time to death or distant metastases; SAEs, incidence of serious adverse events; AEs, incidence of adverse events; DFS, disease-free survival.

found that before treatment, the higher the level of PD-L1 expression, the more effective. Compared to the patients whose PD-L1 $>1 \%$ and PD-L1 $\leq 1 \%$, the former had the lower survived tumors cells (median $20 \%$ vs $80 \%$ ). ${ }^{7}$ The results have been showed that the dual immunoadjuvant therapy is better than the single immunotherapy.

Through Checkmate 159 study, it has been discovered that the use of PD-1 blockers before surgery can enhance the anti-tumor T cells' response ability, and can also make peripheral blood continuously produce more memoryspecific $\mathrm{CD}^{+} \mathrm{T}$ cells. The anti-tumor activity of $\mathrm{T}$ cells continues to destroy cancer cells, thereby potentially eliminating micro-metastatic cancer that might cause recurrence after surgery. Data from many other clinical studies show that nivolumab immunoadjuvant therapy will not affect the operation time, which is expected to change the traditional treatment of lung cancer. The application of neoadjuvant immunotherapy+chemotherapy treatment will enable many patients to shrink tumors before surgery, reduce the difficulty of surgery, and even allow some locally advanced patients who could not be operated to obtain the chances for surgery. In addition, immunotherapy helps to establish immune recognition of tumor cells by itself. Post-operative T cells will identify and attack tumor cells, which helps to eliminate micrometastases and reduce the risk of recurrence. These advantages will greatly improve the cure rate and longterm survival rate of lung cancer. It is worth noting that the median age of people diagnosed with NSCLC is 70 years old, and a considerable number of patients have a history of smoking or other basic diseases. Neoadjuvant immunotherapy gives these patients time to quit smoking. Better management of underlying diseases is a key intervention to reduce risks and create opportunities for intraoperative safety and postoperative recovery. Compared with other therapies, after receiving neoadjuvant immunotherapy, clinicians can evaluate the comprehensive treatment effect based on the pathology of the removed tumor, and determine the follow-up treatment plan accordingly.

However, there is currently no uniform usage of the neoadjuvant immunotherapy cycle. Most clinical trials use 2-3 cycle before and after operation. The efficacy judgement, the prescription for post-operation, and withdrawal criteria are still under discussion. In view of the above problems, in order to better use nivolumab in clinical practice, a large samples of evidence support is still needed. Fortunately, some large-scale new auxiliary studies of nivolumab-related immunity are currently underway (Table 1). We believe these trials will bring surprises.

In the safety analysis, nivolumab-related adverse events are common and relatively well tolerated. In a retrospective study, ${ }^{8}$ scholars found that when using nivolumab in the treatment of NSCLC, patients with immune-related adverse reactions had a better prognosis. That means immune-related adverse reactions may become a predicator of the effect of immunotherapy. Although the incidence of adverse reactions caused by immunotherapy is relatively low, it involves multiple organs and systems throughout the body. Clinicians should inform patients as soon as possible during treatment, only by detecting and treating early, the adverse reactions can be prevented and controlled.

Data from some trials and meta-analyses indicated that $^{9,10}$ there was no significant difference in the perioperative complication rate and postoperative mortality rate between patients receiving neoadjuvant chemotherapy and those undergoing direct surgery.

In the field of immunotherapy, the PD-1/the PD-L1 expression level, tumor mutation burden (TMB) and other indicators of efficacy are commonly used to predict curative effect. The results of the Checkmate 017 study showed that the expression status of PD-L1 was not significantly correlated with the prognosis of squamous cell 
carcinoma patients. $^{11}$ However, in the Checkmate057 study, the benefit of patients with non-squamous carcinoma was related to the expression of PD-L1, ${ }^{11}$ and the patient's remission rate was proportional to the level of PD-L1 expression: among patients with tumors expressing PD-L1, patients with PD-L1 $>5$ had an ORR of $36 \%$ and PFS was 18.1 months, while patients with PD-L $1<5$ had an ORR of $10 \%$ and PFS was only 9.7 months. A summary analysis ${ }^{12}$ showed that patients with high expression of PD-L1 had a higher remission rate (RR:2.19) and a longer progression-free survival (HR:0.69), the overall survival rate was higher (HR:0.77). It is foreseeable that future clinical trials may tend to recruit patients with higher expression levels of PD-1/PD-L1.

\section{Conclusions}

In our report, we demonstrated the efficacy of neoadjuvant nivolumab in a patient with unresectable NSCLC. Due to environmental and tobacco reasons, the number of people suffering from lung cancer is increasing. Clinical studies have shown that compared with the same therapy given in an adjuvant setting, the neoadjuvant drug administration of nivolumab has an improved survival rate and enhanced anti-tumor immune response. In recent years, the rapid progression of immunotherapy has broken through the bottleneck of cytotoxicity-based chemotherapy in wild-type non-small cell lung cancer, and has improved the prognosis and life quality for patients. ICIs have reshaped the management of NSCLC. Tumor immunotherapy represented by nivolumab is an epoch-making revolutionary measure. It has been proven to be superior to chemotherapy in a number of clinical trials. This achievement has breakthrough significance. However, there are still some questions to be resolved. How to choose the best target group? How to combine with other treatment methods to benefit patients more? Are the evaluation criteria consistent after immunotherapy? In our case, the patient had high PD-L1 expression and achieved pCR after treatment. Can pCR be used as an indicator of clinical endpoints? These problems need to be resolved urgently. This is inseparable from the joint participation and dedication of tens of thousands of scientists, medical staff, and patients, and requires a lot of medical evidence. The concerted efforts of all of us have promoted new breakthroughs in the field of anti-cancer. We have reasons to believe that immunotherapy will make more progress in the future, enabling patients to obtain a higher quality of life while prolonging their survival period.

\section{Statement of Ethics}

Written informed consent was obtained from the patient for the publication of both the case report and its accompanying images. This is a retrospective case report and institutional approval was not needed.

\section{Disclosure}

The authors report no conflicts of interest in this work.

\section{References}

1. Provencio-Pulla M, Nadal-Alforja E, Cobo M, et al. Neoadjuvant chemo/immunotherapy for the treatment of stages IIIA resectable non-small cell lung cancer (NSCLC): a phase II multicenter exploratory study-NADIM study-SLCG. $J$ Clin Oncol. 2018;36 (15_suppl):8521.

2. Forde PM, Chaft JE, Smith KN, et al. Neoadjuvant PD-1 blockade in resectable lung cancer. N Engl J Med. 2018;378(21):1976-1986. doi:10.1056/NEJMoa1716078

3. Reuss JE, Smith KN, Anagnostou V, et al. Neoadjuvant nivolumab in resectable non-small cell lung cancer: extended follow-up and molecular markers of response. J Clin Oncol. 2019;37(15_suppl):8524. doi:10.1200/JCO.2019.37.15_suppl.8524

4. Provencio M, Nadal E, Insa A, et al. OA13.05 NADIM Study: updated clinical research and outcomes. J Thorac Oncol. 2019;14 (10):S241. doi:10.1016/j.jtho.2019.08.480

5. Provencio M. ES02. 04 concurrent, sequential and combination immunotherapy regimens in LA-NSCLC. J Thorac Oncol. 2019;14 (10):S17-S18.

6. Provencio M, Nadal E, Insa A, et al. Neoadjuvant chemoimmunotherapy for the treatment of stage IIIA resectable non-smallcell lung cancer (NSCLC): a phase II multicenter exploratory studyfinal data of patients who underwent surgical assessment. J Clin Oncol. 2019;37(15_suppl):8509. doi:10.1200/JCO.2019.37.15_suppl.8509

7. Cascone T, William WN, Weissferdt A, et al. Neoadjuvant nivolumab (N) or nivolumab plus ipilimumab (NI) for resectable non-small cell lung cancer (NSCLC): clinical and correlative results from the NEOSTAR study. J Clin Oncol. 2019;37:8504.

8. Haratani K, Hayashi H, Chiba Y, et al. Association of immune-related adverse events with nivolumab efficacy in non-small-cell lung cancer. JAMA Oncol. 2018;4(3):374.

9. NSCLC Meta-analysis Collaborative Group. Preoperative chemotherapy for non-small-cell lung cancer: a systematic review and metaanalysis of individual participant data. Lancet. 2014;383(9928):15611571.

10. Blumenthal GM, Bunn PA, Chaft JE, et al. Current status and future perspectives on neoadjuvant therapy in lung cancer. $J$ Thorac Oncol. 2018;13(12):1818-1831.

11. Borghaei H, Paz-Ares L, Horn L, et al. Nivolumab versus docetaxel in advanced nonsquamous non-small-cell lung cancer. $N$ Engl J Med. 2015;373(17):1627-1639.

12. Aguiar PN, De Mello RA, Hall P, et al. PD-L1 expression as a predictive biomarker in advanced non-small-cell lung cancer: updated survival data. Immunotherapy. 2017;9(6):499-506. doi:10.2217/imt-2016-0150 


\section{Publish your work in this journal}

OncoTargets and Therapy is an international, peer-reviewed, open access journal focusing on the pathological basis of all cancers, potential targets for therapy and treatment protocols employed to improve the management of cancer patients. The journal also focuses on the impact of management programs and new therapeutic

Submit your manuscript here: https://www.dovepress.com/oncotargets-and-therapy-journal agents and protocols on patient perspectives such as quality of life, adherence and satisfaction. The manuscript management system is completely online and includes a very quick and fair peer-review system, which is all easy to use. Visit http://www.dovepress.com/ testimonials.php to read real quotes from published authors. 